\title{
Sequence-based identification of Anopheles species in eastern Ethiopia
}

\author{
Tamar E. Carter ${ }^{1 *}$ D , Solomon Yared ${ }^{2}$, Shantoy Hansel ${ }^{3}$, Karen Lopez ${ }^{3}$ and Daniel Janies ${ }^{3}$
}

\begin{abstract}
Background: The recent finding of a typically non-African Anopheles species in eastern Ethiopia emphasizes the need for detailed species identification and characterization for effective malaria vector surveillance. Molecular approaches increase the accuracy and interoperability of vector surveillance data. To develop effective molecular assays for Anopheles identification, it is important to evaluate different genetic loci for the ability to characterize species and population level variation. Here the utility of the internal transcribed spacer 2 (ITS2) and cytochrome oxidase I (COI) loci for detection of Anopheles species from understudied regions of eastern Ethiopia was investigated.

Methods: Adult mosquitoes were collected from the Harewe locality (east) and Meki (east central) Ethiopia. PCR and Sanger sequencing were performed for portions of the ITS2 and COI loci. Both NCBI's Basic Local Alignment Search tool (BLAST) and phylogenetic analysis using a maximum-likelihood approach were performed to identify species of Anopheles specimens.

Results: Two species from the east Ethiopian collection, Anopheles arabiensis and Anopheles pretoriensis were identified. Analyses of ITS2 locus resulted in delineation of both species. In contrast, analysis of COI locus could not be used to delineate An. arabiensis from other taxa in Anopheles gambiae complex, but could distinguish An. pretoriensis sequences from sister taxa.

Conclusion: The lack of clarity from COI sequence analysis highlights potential challenges of species identification within species complexes. These results provide supporting data for the development of molecular assays for delineation of Anopheles in east Ethiopia.
\end{abstract}

Keywords: Anopheles arabiensis, Malaria, Phylogenetics, Internal transcribed spacer 2, Cytochrome oxidase subunit I, Horn of Africa

\section{Background}

Over 1.5 million cases of malaria were reported in Ethiopia in 2017 [1]. While strides to control the transmission of malaria likely contributed to the reduction in overall mortality and incidence over the last several decades [2], continued understanding of the mosquito vector populations are needed for improved targeted interventions [3]. In east Ethiopia, Anopheles species are still being uncovered. A recent study revealed the presence of Anopheles stephensi, a malaria vector species typically only seen east of the Red Sea [4]. Historically, the number of malaria

\footnotetext{
*Correspondence: tamar_carter@baylor.edu

${ }^{1}$ Department of Biology, Baylor University, Waco, TX, USA

Full list of author information is available at the end of the article
}

cases have been low in this region, but the presence of potential malaria vectors and recent reports of sporadic malaria outbreaks warrant further investigation of vector populations.

Due to the global variation of Anopheles species and populations, it is vital to evaluate techniques specific to east Ethiopia to identify various Anopheles species [3, 5]. Once a technique is validated the diversity and distribution of various Anopheles species can be accurately determined and the proper intervention implemented. In Ethiopia, much of the mosquito surveillance and identification is conducted using mosquito morphology, e.g. [6-9]. Morphological identification can be tedious when processing many specimens and comes with risk for misidentification of species not previously encountered

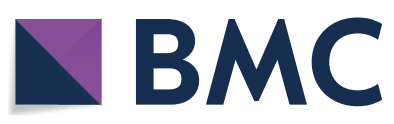

(c) The Author(s) 2019. This article is distributed under the terms of the Creative Commons Attribution 4.0 International License (http://creativecommons.org/licenses/by/4.0/), which permits unrestricted use, distribution, and reproduction in any medium, provided you give appropriate credit to the original author(s) and the source, provide a link to the Creative Commons license, and indicate if changes were made. The Creative Commons Public Domain Dedication waiver (http://creativecommons.org/ publicdomain/zero/1.0/) applies to the data made available in this article, unless otherwise stated. 
and potentially cryptic species [5]. Genetic analysis can be employed as a high-throughput approach to identify mosquito species. Moreover, because the DNA data are interoperable with previous DNA records and often is linked to rich metadata on location and date of isolation, one can build information about population structure and movement of vector species to improve our understanding of the spatial epidemiology of malaria. Analysis of the nuclear internal transcribed spacer 2 (ITS2) and mitochondrial cytochrome oxidase I (COI, also called CO1, COX1) loci have served as the basis of species identification assays that use allele-specific PCR amplification $[10,11]$, restriction enzyme digestions [12], or genetic sequencing-based assays $[4,5,13]$. Identifying the correct locus or loci for the basis of species or population-level analysis is important. Previous studies have highlighted how the analysis of the COI gene poses challenges for discriminating between closely related species such as those which belong to a species complex (for review see Beebe et al. [14]). In this study, the ITS2 and the COI loci were sequenced and analysed for species identification in Anopheles specimens collected in two sites in east Ethiopia to evaluate the potential of these loci for identification of east Ethiopian Anopheles.

\section{Methods}

\section{Study locations}

Mosquito specimens were collected during four collections from two sites, Harewe locality and Meki, in east Ethiopia (Table 1). These regions were selected because malaria cases have been reported there in recent years $[15,16]$. The Harewe locality is in the Harari Region at $9^{\circ} 16^{\prime} \mathrm{N}$ latitude and $42^{\circ} 10^{\prime} \mathrm{E}$ longitude, $15 \mathrm{~km}$ from Harar city. Harewe has a mountainous landscape with an elevation of $1552 \mathrm{~m}$ above sea level. A small river runs across the Harewe valley between the mountains and is suspected to be a breeding habitat of Anopheles mosquitoes. Meki is a town in east-central Ethiopia, $130 \mathrm{~km}$ from

Table 1 Specimen collection sites, dates, GPS coordinates, and quantities

\begin{tabular}{llll}
\hline Collection site & $\begin{array}{l}\text { Date } \\
\text { of collection }\end{array}$ & GPS coordinates & $\begin{array}{l}\text { Number } \\
\text { of mosquitoes }\end{array}$ \\
\hline Harar & $\begin{array}{l}\text { November 21, } \\
2016\end{array}$ & $\begin{array}{c}9.3126^{\circ} \mathrm{N}, \\
42.1227^{\circ} \mathrm{E}\end{array}$ & 22 \\
Harar & July 21, 2017 & $\begin{array}{c}9.3126^{\circ} \mathrm{N}, \\
42.1227^{\circ} \mathrm{E}\end{array}$ & 8 \\
& & $\begin{array}{c}8.1552^{\circ} \mathrm{N}, \\
38.8258^{\circ} \mathrm{E}\end{array}$ & 31 \\
Meki & July 28, 2017 & $9.3126^{\circ} \mathrm{N}$, \\
& $42.1227^{\circ} \mathrm{E}$ & 32 \\
Harar & August 19, 2017 & 93 \\
Total & & & 9 \\
\hline
\end{tabular}

Addis Ababa. Meki is located in the East Shewa Zone of the Oromia Region in the middle of the Rift Valley area, at latitude $8^{\circ} 9^{\prime} \mathrm{N}$ and longitude $38^{\circ} 49^{\prime} \mathrm{E}$ with an elevation of $1636 \mathrm{~m}$ above sea level. Meki has a tropical climate and is surrounded by lakes. Small-scale irrigation is practiced by the community in Meki.

\section{Sample collection}

The three collections in the Harewe locality took place in November 2016, July 2017, and August 2017. The Meki collection took place in August 2017. Mosquitoes were collected indoors and outdoors from 6:00 pm to 6:00 am from each selected areas using standard CDC light traps (John W. Hock, Gainesville, FL, USA). Indoor traps were hung from the ceiling or from roof supports at the foot end of beds where people sleep at night. Outdoor collection traps were placed close to breeding habitats and the body of the trap was suspended about $1.5 \mathrm{~m}$ from the ground. A total of $16 \mathrm{CDC}$ light traps were deployed for collection of mosquitoes in each study area.

Collected mosquitoes were kept in paper cups and brought to the field laboratory for identification. At the laboratory, mosquitoes were anesthetized with chloroform and all adult mosquitoes were counted and identified, under steromicroscopes, to at least genus level based on a morphological key [17].

\section{Amplification and sequencing}

Molecular analysis was performed on collected Anopheles mosquitoes to determine species and characterize the genetic variation within species. Species identification was completed using amplification of two genes: ITS2 and COI. Legs were used as DNA templates for PCR. For ITS2 amplification, PCR amplifications were performed as described previously [4] using the following: primers 5.8S ATCACTCGGCTCGTGGATCG and 28S ATGCTT AAATTTAGGGGGTAGTC for ITS2 [11]. Starting reagents concentrations were as follows: $10 \mathrm{mM}$ for each primer, 2X Promega GoTAQ HotStart master mix (Promega, Madison, Wisconsin), and water for a total reaction volume of $25 \mu \mathrm{l}$. PCR amplifications were performed with the following temperature cycling: $95^{\circ} \mathrm{C}$ for $2 \mathrm{~min}$, 30 cycles of $95^{\circ} \mathrm{C}$ at $30 \mathrm{~s}, 50^{\circ} \mathrm{C}$ at $30 \mathrm{~s}, 72^{\circ} \mathrm{C}$ at $1 \mathrm{~min}$, and final extension of $72{ }^{\circ} \mathrm{C}$ at $5 \mathrm{~min}$. The protocol for COI was the same as ITS2 protocol except that the primers used were LCO1490F GGTCAACAAATCATAAAGATA TTGG and HCO2198R TAAACTTCAGGGTGACCA AAAAATCA for COI [18]. Temperature cycling for COI PCR was as follows: $95^{\circ} \mathrm{C}$ at $1 \mathrm{~min}, 30$ cycles of $95^{\circ} \mathrm{C}$ for $30 \mathrm{~s}, 48^{\circ} \mathrm{C}$ for $30 \mathrm{~s}, 72{ }^{\circ} \mathrm{C}$ for $1 \mathrm{~min}$, with a final extension of $72{ }^{\circ} \mathrm{C}$ for $10 \mathrm{~min}$. For both ITS2 and COI, eight microliters of PCR product were run on $2 \%$ agarose gel for $1 \mathrm{~h}$ at $100 \mathrm{~V}$ to confirm successful PCR products which 
were then cleaned using ExoSAP. PCR products were sequenced using Sanger technology with ABI BigDyeTM Terminator v3.1 chemistry (Thermofisher, Santa Clara, CA) according to manufacturer recommendations and run on a 3130 Genetic Analyzer (Thermo Fisher, Santa Clara, CA).

\section{Sequence analysis for species identification}

Sequences were cleaned and analysed using CodonCode Aligner Program V. 6.0.2 (CodonCode Corporation, Centerville, MA). ITS2 and COI sequences from Anopheles specimens were submitted as queries to the National Center for Biotechnology Information's (NCBI) web-based Basic Local Alignment Search Tool (BLAST) [19] against the nucleotide collection in Genbank under default parameters [max High-scoring Segment Pairs (HSP) 250, expect threshold 10, word size 28, optimized for highly similar hits, not specific to any organism]. The Anopheles subject sequences from NCBI that formed HSP with the queries were identified.

Phylogenetic analyses of ITS2 and COI were employed to search for sister taxon relationships between isolates of Anopheles from east Ethiopia and voucher specimens from Anopheles with orthologous sequence data stored in NCBI. Anopheles sequences from east Ethiopia and closest sequence hits in BLAST that had more than 85\% sequence coverage were combined into datasets for COI and ITS2 separately. In some cases, there were multiple sequences from the same location and study. In these instances, only representative sequences were taken from those population sets. Alignments were created with MAFFT version 7 under default parameters [18] and ragged ends were trimmed using Mesquite 3.51 [20]. Phylogenetic relationships with the Ethiopian Anopheles sequences and Anopheles sequences from NCBI were inferred using RAxML [21] which is based on a maximum likelihood (ML) approach. The GTRGAMMA option that uses GTR model of nucleotide substitution with gamma model of rate of heterogeneity was applied. Both 100 and 1000 replicates were completed with the strategy searching for the heuristically-best-scoring tree and bootstrap analysis in one run. Best scoring trees under ML with bootstrap values from RAxML were viewed and rooted under the outgroup criterion in FigTree [22] for each locus. Outgroups were chosen based on availability of sequence data for each locus, overall coverage, and its use in previous phylogenetic analyses. For the COI analysis, Anopheles implexus sequence was used as an outgroup based on sequence availability and use in similar analyses of Anopheles species [4]. For the ITS2, a different species, Anopheles christyi, was used as an outgroup primarily because An. implexus sequence was not available.
Compatible An. christyi ITS2 sequence was available and this species had been used in a similar analysis [23].

\section{Results \\ ITS2 sequence analysis}

The ITS2 sequences were analysed for a subset of samples from each collection from Ethiopia $(n=82)$. All Anopheles gambiae complex specimens from this collection in Ethiopia were identical for ITS2 sequences. When the consensus ITS2 sequence from Ethiopia were searched against NCBI with BLAST, specimens from Ethiopia formed HSP with 99\% identity for Anopheles arabiensis.

ITS2 sequence data from eight non-Anopheles gambiae complex specimens from Ethiopia were generated and all sequences were identical. Based on BLAST against NCBI sequences, these ITS2 sequences from Ethiopia formed HSP with 99\% identity for Anopheles pretoriensis.

\section{COI sequence analysis}

A subset of the samples from each collection from Ethiopia were chosen for PCR amplification and sequencing of a portion the COI gene $(n=37)$. Sequences were cleaned and trimmed and submitted as queries to NCBI's BLAST. Of the 37 sequences from Ethiopian specimens, 29 formed HSP with 99\% identity for both An. arabiensis and An. gambiae sequences in the NCBI database.

These 29 specimens for which COI sequences had ambiguous HSP with respect to species had coinciding ITS2 sequences (see above) that confirmed their identity as $A n$. arabiensis. The number of unique COI sequences (haplotypes) was determined. COI sequences with at least $578 \mathrm{bp}(\mathrm{n}=20)$ of readable sequence data revealed 12 different haplotypes.

The remaining eight sequences from Ethiopian specimens formed HSP with NCBI data for within 99\% for An. pretoriensis sequence vouchers. These eight Ethiopian specimens had coinciding ITS2 data that confirmed their identity as An. pretoriensis. Six of these specimens had at least $611 \mathrm{bp}$ of readable sequence and each had a unique COI haplotype.

\section{Phylogenetic analysis for further species differentiation}

To confirm the results of the ITS2 BLAST analysis identifying $A n$. arabiensis specimens, phylogenetic analysis was performed with the closest hits in Genbank within the An. gambiae complex (Additional file 1: Table S1a, Additional file 2). The analysis for 100 and 1000 bootstrap replicates produced similar final ML likelihood scores (both $=-1110.7$ ). Figure 1 shows the tree for the 100 bootstrap replicates. The An. arabiensis sequences from NCBI formed a clade that included the Ethiopian specimens with bootstrap support of 99\% (Fig. 1). This An. 


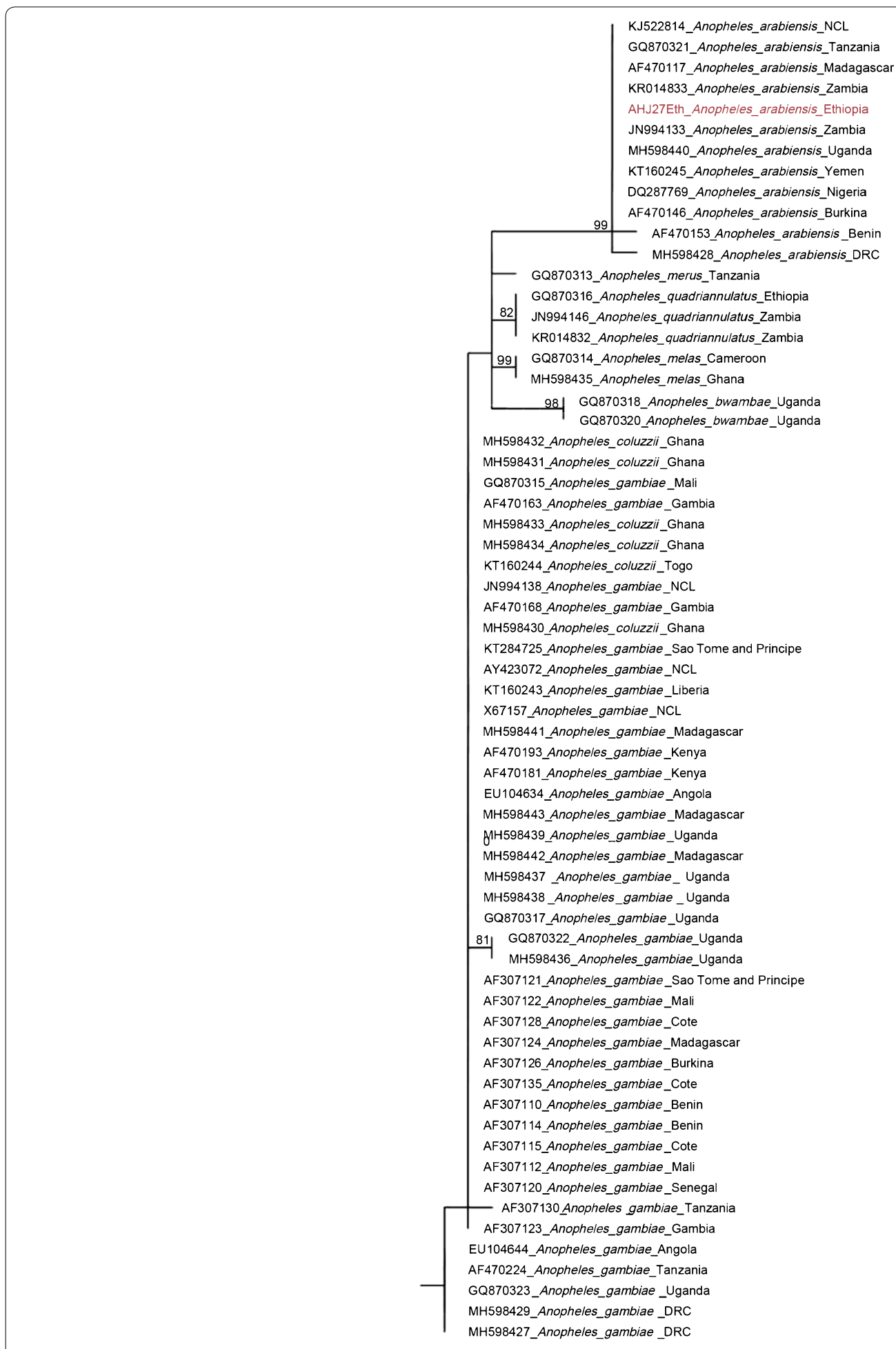

\subsection{3}

Fig. 1 Maximum-likelihood tree of Anopheles ITS2 sequences. Analysis based on a 465 base pair sequence of the locus. The taxon in red is a representative specimen collected in Ethiopia from the present study (all Ethiopian ITS2 sequences were identical). Tree includes An. gambiae complex sequences taken from NCBI's Genbank. Bootstrap values 70 and higher are shown. Outgroup (Anopheles christyi) not shown. Final ML Optimization Likelihood: - 1110.705351 
arabiensis clade was distinct from all other An. gambiae complex species. Anopheles pretoriensis and other more distant Anopheles taxa could not be included in these analyses due to large deleted regions in the ITS2 of these taxa. However, BLAST analysis was sufficient to determine An. pretoriensis identification.

Phylogenetic analysis was also completed using the COI sequences. A collection of sequences from the $A n$. gambiae complex and representative sequences from other Anopheles species (including the Ethiopian An. stephensi sequence) were included in the phylogenetic analysis (Additional file 1: Table S1b, Additional file 3). The analysis for 100 and 1000 bootstrap replicates produced similar final ML likelihood scores (both $=-2668.8$ ). Figure 2 shows the tree for the 100 bootstrap replicates. Based on the phylogenetic analysis of COI, the An. arabiensis Ethiopian sequences fell within the An. gambiae complex clade (Fig. 2, bootstrap $=100$ ) to the exclusion of An. pretoriensis and other species outside the An. gambiae complex. Within An. gambiae complex clade, the $A n$. arabiensis and An. gambiae sequences could not be differentiated with these data. In addition, no differentiation was observed between the Harewe locality and Meki Ethiopian specimens. The An. pretoriensis specimens from NCBI and from Ethiopia formed a clade separate from other Anopheles species, with some bootstrap support $=100$.

\section{Discussion}

ITS2 and COI sequence variation in Anopheles showed different levels of success in identifying Anopheles species found in east Ethiopia. Both loci confidently distinguished specimens belonging to the An. gambiae complex species from those that do not (i.e. An. pretoriensis). ITS2 provides further resolving power to differentiate An. arabiensis specimens from other An. gambiae complex specimens.

For the COI data, An. arabiensis and An. gambiae specimens formed a clade that could not be distinguished as species level subclades (Fig. 1). Previous studies have shown similar results with mtDNA [23, 24]. Anopheles arabiensis and An. gambiae are closely related species so the similarity in sequence may be due to incomplete evolutionary sorting or hybridization between species $[14,23-25]$ that reduces power to distinguish species. In contrast, COI sequences proved to be very useful for the species identification of An. stephensi in Ethiopia [4].

There was interest in whether within species differentiation could be observed for the Ethiopian specimens. While initial sequence analysis showed substantial haplotype variation in the COI locus within the Ethiopian sequences, phylogenetic analysis did not reveal any within-species differentiation, geographic or otherwise for the confirmed An. arabiensis sequences. There was some within-species differentiation for An. pretoriensis sequences. COI locus revealed some differentiation within the An. stephensi grouping [4]. Taken together, these results indicate that $\mathrm{COI}$ has some utility related to within species differentiation for some Anopheles species, but not for others.

The results presented in this study confirm the presence of An. pretoriensis in the Harewe locality. This species has been observed in other parts of Ethiopia including the southwest and northern regions [26, 27]. Anopheles pretorienis has not been considered a strong vector of malaria. Indeed, blood meal analysis of two blood-fed An. pretoriensis from this study indicated only bovine feeding (data not shown). However, a recent study showed An. pretoriensis was positive for Plasmodium falciparum in Zambia, suggesting it is important to understand the distribution of this species in Ethiopia as a potential vector [5]. Additionally, subspecies differentiation for one An. pretoriensis specimen in the COI analysis was observed (Fig. 2, bootstrap $=74 \%$ ). Questions remain whether there is significant evolutionary divergence within the $A n$. pretoriensis species and if it is associated with vector competence.

These findings have implications for the design of molecular assays to differentiate Anopheles species in east Ethiopia. ITS2 has proven to be a more useful sequence-based approach to determine species using simple BLAST analysis. Phylogenetic analysis of the COI can be useful for sequence-based analysis of some Anopheles species found in east Ethiopia, but not for members of the An. gambiae complex. One approach that may improve molecular species identification would be to combine the two loci into a single analysis. Previous studies have employed multiple loci $[13,24]$ and require the availability of genomic or coordinating database sequences for both loci from the same specimens representing relevant species and populations. If such sequence data are available, phylogenetic analysis that incorporates genes with various rates of evolution often provides better insight into both between and within species diversity.

\section{Conclusion}

In conclusion, ITS2 and COI vary in their ability to delineate Anopheles species. The results of the COI analysis of An. arabiensis specimens revealed the potential challenge of using just that locus for molecular species identification of within species complexes. The results of this study contribute to development of molecular assays for Anopheles species identification in east Ethiopia. 


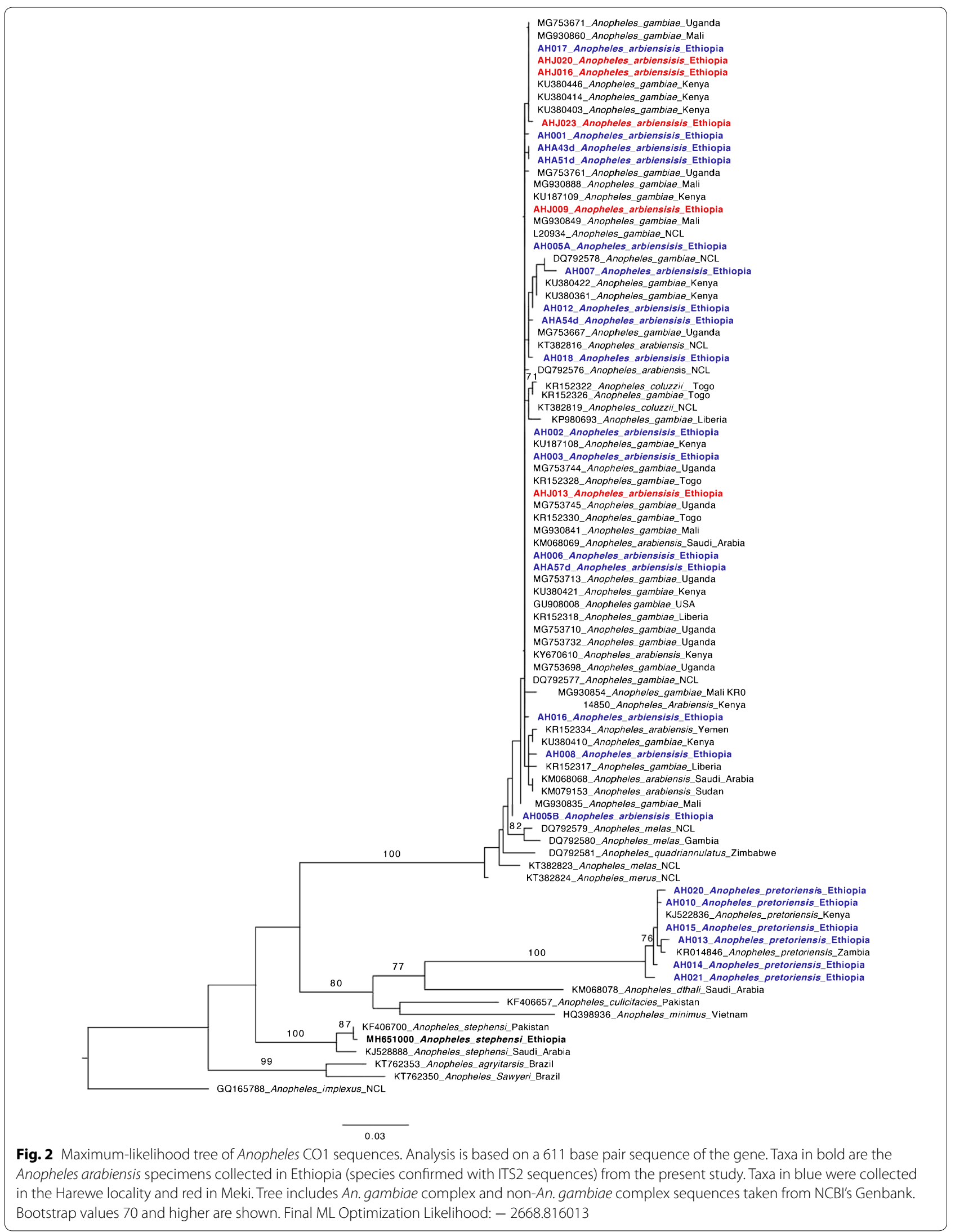




\section{Additional files}

Additional file 1. List of sequences from NCBI database used in phylogenetic analysis.

Additional file 2. ITS2 Sequence alignment including Ethiopian and NCBI Genbank sequences.

Additional file 3. COI Sequence alignment including Ethiopian and NCBI Genbank sequences.

\section{Abbreviations}

BLAST: Basic Local Alignment Search Tool; COl: cytochrome c oxidase subunit 1 gene; DNA: deoxyribonucleic acid; HSP: high-scoring segment pairs; ITS2: internal transcribed spacer 2 region; NCBI: National Center of Biotechnology Information; PCR: polymerase chain reaction.

\section{Authors' contributions}

TC: conceptualizing the study, molecular analysis, data analysis, and manuscript preparation; SY: the collection of specimens, conceptualizing the study, and manuscript preparation; $\mathrm{SH}$ : molecular analysis and sequence analysis; $\mathrm{KL}$ : molecular analysis; and DJ: conceptualizing the project and manuscript preparation. All authors read and approved the final manuscript.

\section{Author details}

${ }^{1}$ Department of Biology, Baylor University, Waco, TX, USA. ${ }^{2}$ Department of Biology, Jigjiga University, Jigjiga, Ethiopia. ${ }^{3}$ Department of Bioinformatics and Genomics, University of North Carolina at Charlotte, Charlotte, NC, USA.

\section{Acknowledgements}

The authors would like to thank Sae Hee Choi for assistance with data management.

\section{Competing interests}

The authors declare that they have no competing interests.

\section{Consent for publication}

Not applicable.

\section{Data availability}

The sequences generated and/or analysed during the current study are available in NCBI's Genbank. https://www.ncbi.nlm.nih.gov/genbank/. Genbank accession numbers for Ethiopia sequences generated in this study are MK628480-MK628508.

\section{Ethics approval and consent to participate}

Not applicable.

\section{Funding}

Research reported in this publication was also supported by a UNC Research Opportunities Initiative grant, UNC Charlotte Multicultural Postdoctoral Fellowship from Academic Affairs, and Baylor University.

\section{Publisher's Note}

Springer Nature remains neutral with regard to jurisdictional claims in published maps and institutional affiliations.

Received: 24 January 2019 Accepted: 4 April 2019

Published online: 16 April 2019

\section{References}

1. WHO. World malaria report 2017. Geneva: World Health Organization; 2018.

2. Deribew A, Dejene T, Kebede B, Tessema GA, Melaku YA, Misganaw A, et al. Incidence, prevalence and mortality rates of malaria in Ethiopia from 1990 to 2015: analysis of the global burden of diseases 2015. Malar J. 2017;16:271.

3. Animut A, Lindtjorn B. Use of epidemiological and entomological tools in the control and elimination of malaria in Ethiopia. Malar J. 2018;17:26.
4. Carter TE, Yared S, Gebresilassie A, Bonnell V, Damodaran L, Lopez K, et al. First detection of Anopheles stephensi Liston, 1901 (Diptera: culicidae) in Ethiopia using molecular and morphological approaches. Acta Trop. 2018;188:180-6.

5. Lobo NF, St Laurent B, Sikaala CH, Hamainza B, Chanda J, Chinula D, et al. Unexpected diversity of Anopheles species in Eastern Zambia: implications for evaluating vector behavior and interventions using molecular tools. Sci Rep. 2015;5:17952.

6. Degefa T, Zeynudin A, Godesso A, Michael YH, Eba K, Zemene E, et al. Malaria incidence and assessment of entomological indices among resettled communities in Ethiopia: a longitudinal study. Malar J. 2015;14:24.

7. Massebo F, Balkew M, Gebre-Michael T, Lindtjorn B. Zoophagic behaviour of anopheline mosquitoes in southwest Ethiopia: opportunity for malaria vector control. Parasit Vectors. 2015;8:645

8. Kenea O, Balkew M, Tekie H, Gebre-Michael T, Deressa W, Loha E, et al. Human-biting activities of Anopheles species in south-central Ethiopia. Parasit Vectors. 2016;9:527.

9. Animut A, Negash Y. Dry season occurrence of Anopheles mosquitoes and implications in Jabi Tehnan District, West Gojjam Zone Ethiopia. Malar J. 2018;17:445.

10. Koekemoer LL, Kamau L, Hunt RH, Coetzee M. A cocktail polymerase chain reaction assay to identify members of the Anopheles funestus (Diptera: Culicidae) group. Am J Trop Med Hyg. 2002;66:804-11.

11. Djadid ND, Gholizadeh S, Aghajari M, Zehi AH, Raeisi A, Zakeri S. Genetic analysis of rDNA-ITS2 and RAPD loci in field populations of the malaria vector, Anopheles stephensi (Diptera: Culicidae): implications for the control program in Iran. Acta Trop. 2006;97:65-74.

12. Goswami G, Raghavendra K, Nanda N, Gakhar SK, Subbarao SK. PCR-RFLP of mitochondrial cytochrome oxidase subunit II and ITS2 of ribosomal DNA: markers for the identification of members of the Anopheles culicifacies complex (Diptera: Culicidae). Acta Trop. 2005:95:92-9.

13. Bourke BP, Oliveira TP, Suesdek L, Bergo ES, Sallum MA. A multi-locus approach to barcoding in the Anopheles strodei subgroup (Diptera: Culicidae). Parasit Vectors. 2013;6:111.

14. Beebe NW. DNA barcoding mosquitoes: advice for potential prospectors. Parasitology. 2018;145:622-33.

15. Tadesse F, Fogarty AW, Deressa W. Prevalence and associated risk factors of malaria among adults in East Shewa Zone of Oromia Regional State, Ethiopia: a cross-sectional study. BMC Public Health. 2017;18:25.

16. Ethiopia National Malaria Indicator Survey 2015. Ethiopian Public Health Institute; 2016

17. Gillies MT, Coetzee, M. A supplement to the Anophelinae of Africa South of the Sahara. Publ S Afr Inst Med Res. 1987;55:1-43.

18. Folmer O, Black M, Hoeh W, Lutz R, Vrijenhoek R. DNA primers for amplification of mitochondrial cytochrome c oxidase subunit I from diverse metazoan invertebrates. Mol Mar Biol Biotechnol. 1994:3:294-9.

19. Altschul SF, Gish W, Miller W, Myers EW, Lipman DJ. Basic local alignment search tool. J Mol Biol. 1990;215:403-10.

20. Maddison WP, Maddison DR. Mesquite: a modular system for evolutionary analysis. 3.51 ed. 2018.

21. Stamatakis A. RAxML version 8: a tool for phylogenetic analysis and postanalysis of large phylogenies. Bioinformatics. 2014;30:1312-3.

22. Rambaut A. FigTree, a graphical viewer of phylogenetic trees. 2007.

23. Fontaine MC, Pease JB, Steele A, Waterhouse RM, Neafsey DE, Sharakhov $\mathrm{IV}$, et al. Mosquito genomics. Extensive introgression in a malaria vector species complex revealed by phylogenomics. Science. 2015;347:1258524.

24. Besansky NJ, Powell JR, Caccone A, Hamm DM, Scott JA, Collins FH. Molecular phylogeny of the Anopheles gambiae complex suggests genetic introgression between principal malaria vectors. Proc Natl Acad Sci U S A. 1994:91:6885-8.

25. Donnelly MJ, Pinto J, Girod R, Besansky NJ, Lehmann T. Revisiting the role of introgression vs shared ancestral polymorphisms as key processes shaping genetic diversity in the recently separated sibling species of the Anopheles gambiae complex. Heredity (Edinb). 2004;92:61-8.

26. Kindu M, Aklilu E, Balkew M, Gebre-Michael T. Study on the species composition and ecology of anophelines in Addis Zemen, South Gondar Ethiopia. Parasit Vectors. 2018;11:215.

27. Massebo F, Balkew M, Gebre-Michael T, Lindtjorn B. Entomologic inoculation rates of Anopheles arabiensis in southwestern Ethiopia. Am J Trop Med Hyg. 2013;89:466-73. 